\title{
Novos registros de briófitas para Pernambuco, Brasil ${ }^{1}$
}

\author{
Shirley Rangel Germano ${ }^{2,3}$ e Kátia Cavalcanti Pôrto
}

Recebido em 13/02/2003. Aceito em 26/09/2003

RESUMO - (Novos registros de briófitas para Pernambuco, Brasil). Os novos registros são provenientes de um remanescente de

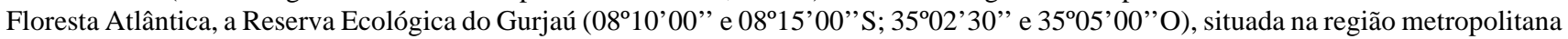
do Recife, Pernambuco. São oito espécies de Lejeuneaceae: Archilejeunea auberiana (Mont.) A. Evans, Cololejeunea cardiocarpa (Mont.) A. Evans, Colura greig-smithii Jovet-Ast, Diplasiolejeunea cobrensis Gottsche ex Steph., Harpalejeunea stricta (Lindenb. \& Gottsche) Steph., Lejeunea caespitosa Lindenb., L. monimiae (Steph.) Steph., L. quinqueumbonata Spruce, e uma de Bryaceae: Bryum pabstianum C. Muell. São apresentadas ilustrações, comentários taxonômicos e ecológicos, e distribuição geográfica das espécies no Brasil.

Palavras-chave: hepáticas, musgos, Floresta Atlântica, Pernambuco

\begin{abstract}
New records of bryophytes for the Pernambuco State, Brazil). The new records were found in an Atlantic Forest remnant (tropical lowland rainforest), Reserva Ecológica do Gurjaú ( $08^{\circ} 10^{\prime} 00^{\prime \prime}$ and $08^{\circ} 15^{\prime} 00^{\prime}$ 'S; 3502'30” and 35'05'00”'W), situated in the metropolitan area of Recife, Pernambuco State, Northeastern Brazil. The new occurrence corresponds to eight species of Lejeuneaceae: Archilejeunea auberiana (Mont.) A. Evans, Cololejeunea cardiocarpa (Mont.) A. Evans, Colura greig-smithii Jovet-Ast, Diplasiolejeunea cobrensis Gottsche ex Steph., Harpalejeunea stricta (Lindenb. \& Gottsche) Steph., Lejeunea caespitosa Lindenb., L. monimiae (Steph.) Steph., L. quinqueumbonata Spruce, and a Bryaceae: Bryum pabstianum C. Muell. Illustrations, floristic and ecological comments, and geographical distribution in Brazil are given for each species.
\end{abstract}

Key words: liverworts, mosses, tropical lowland rainforest, Pernambuco

\section{Introdução}

Em histórico sobre o conhecimento das briófitas do Nordeste do Brasil, Pôrto (1996) apontou Pernambuco e Bahia como os Estados com maior aporte de informações. No que diz respeito particularmente a Pernambuco, diversos trabalhos sistemáticos têm sido desenvolvidos, sobretudo nas duas últimas décadas, o que permitiu a Pôrto \& Germano (2002) compilarem 315 espécies de briófitas para o Estado. Estes valores correspondem a cerca de $10 \%$ do total de espécies referidos para o Brasil por Yano (1996). A maioria dos registros (cerca de 80\%) são provenientes de refúgios de Floresta Atlântica sensu lato, dos quais fazem parte remanescentes costeiros de terras baixas (Pôrto 1990; Pôrto et al. 1993; Germano \& Pôrto 1996; 1998a; 1998b; Sá \& Pôrto 1996), ou submontanas, conhecidas regionalmente como florestas serranas dos brejos de altitude (Yano \& Andrade-Lima 1987; Pôrto 1990; Pôrto et al. 1999; 2000; Valdevino et al. 2002). Nestas formações, as condições ambientais, sobretudo umidade elevada, favorecem o estabelecimento deste grupo de criptógamas.

Durante estudo florístico e ecológico das briófitas, recentemente realizado em um remanescente de Floresta Atlântica, a Reserva Ecológica do Gurjaú, foram registradas 84 espécies desse grupo, das quais nove constituem novas ocorrências para Pernambuco. Neste trabalho é ampliada a distribuição geográfica e são fornecidos dados florísticos e ecológicos das espécies.

\section{Material e métodos}

A Reserva Ecológica do Gurjaú, criada pela Lei 9.989 de 13/01/87, é uma das maiores Unidades de Conservação dentre as 44 reservas florestais de Pernambuco, com 1.362ha. Situa-se na Região Metropolitana do Recife ( $08^{\circ} 10^{\prime} 00^{\prime \prime}-08^{\circ} 15^{\prime} 00^{\prime \prime} \mathrm{S}$; $35^{\circ} 02^{\prime} 30^{\prime}$ ' $-35^{\circ} 05^{\prime} 00^{\prime}$ 'O) e é administrada pela Companhia Pernambucana de Saneamento COMPESA (FIDEM 1993).

\footnotetext{
1 Parte da Tese de Doutorado da primeira Autora; auxílios CAPES, CNPq e WWF

2 Departamento de Botânica, Centro de Ciências Biológicas, Universidade Federal de Pernambuco, Av. Prof. Moraes Rego s/n, Cidade Universitária, CEP 50670-901, Recife, PE, Brasil

3 Autor para correspondência: sgrangel@ufpe.br
} 
As amostras de briófitas foram coletadas nos fragmentos florestais de maior tamanho e melhor estado de conservação, sobre folhas, troncos mortos, base, troncos, ramos e galhos de árvores vivas. Após estudo, os exemplares foram depositados no Herbário da Universidade Federal de Pernambuco (UFP).

As identificações basearam-se nas publicações de Reyes (1982), Tixier (1985), Gradstein (1989; 1994), Heinrichs \& Gradstein (2000), Reiner-Drehwald (2000) e Gradstein \& Costa (2003).

Todas as espécies são ilustradas e apresentadas em ordem alfabética. São fornecidos comentários de relevância para a identificação taxonômica da espécie e notas ecológicas, além da distribuição geográfica no Brasil. Os Estados brasileiros são apresentados conforme sigla oficial.

\section{Resultados e discussão}

Os novos registros pertencem às famílias Lejeuneaceae (oito espécies) e Bryaceae (uma espécie), que são apresentados a seguir:

\section{LEJEUNEACEAE}

Archilejeunea auberiana (Mont.) A. Evans, Bull. Torrey Bot. Club 35: 168. 1908.

Fig. 1-5.

Descrição: Gradstein (1994).

Material examinado: BRASIL. Pernambuco: Cabo de Sto. Agostinho, Reserva Ecológica de Gurjaú, 3/XI/2000, base de tronco vivo, S.R. Germano (UFP 32798); ibid. 3/XI/2000, tronco vivo, S.R. Germano (UFP 32799).

Comentários: espécie próxima à Archilejeunea parviflora (Nees) Schiffn., distinguindo-se desta pelos lóbulos retangulares, grandes, que podem atingir até $1 / 2$ do comprimento do lobo, pelos dois dentes apicais ao invés de um e pelo gametófito de cor verde mais claro. Epífita de troncos, ocorrendo em pequenas populações nos ambientes abertos no interior da mata.

Distribui-se na América Tropical (Gradstein 1994). No Brasil é encontrada nos Estados: AC, AM, PA, PR, RS, SP (Yano 1984; Gradstein 1994; Vital \& Visnadi 1994). Primeiro registro para a região Nordeste.

Cololejeunea cardiocarpa (Mont.) A. Evans, Mem. Torrey Bot. Club 8: 172. 1902.

Fig. 6-10.

Descrição: Tixier (1985).

Material examinado: BRASIL. Pernambuco:
Cabo de Sto. Agostinho, Reserva Ecológica de Gurjaú, 1/XI/2000, folha, S.R. Germano (UFP 31143a).

Comentários: caracteriza-se por apresentar uma camada de células hialinas, em forma de dedos, restritas ao ápice dos filídios. Segundo Schuster (1980), esta é característica marcante para a identificação da espécie, embora nem sempre se apresente bem desenvolvida. Ocasionalmente, uma camada de células hialinas quadráticas ou retangulares pode ocorrer na margem do filídio. Gemas discóides usualmente presentes na superfície do filídio. Epífila, ocorrendo em pequena população, em ambiente semi-aberto no interior da mata.

Pantropical. No Brasil é encontrada nos Estados: AM, PB, PR, ES, RJ, RO, RR, SP (Yano 1984; 1989). Primeiro registro para Pernambuco.

Colura greig-smithii Jovet-Ast, Revue Bryol. Lichénol. 22: 293. 1953.

Fig. 11-14.

Descrição: Jovet-Ast (1953).

Material examinado: BRASIL. Pernambuco: Cabo de Sto. Agostinho, Reserva Ecológica de Gurjaú, 1/XI/2000, folha, S.R. Germano (UFP 31126c; UFP 31128a; UFP 31129d; UFP 31135e; UFP 31149a; UFP 31170b; UFP 31172b).

Comentários: caracteriza-se pelo filídio ovalado a oblongo em forma de saco, com superfície crenulada por células cônicas, especialmente no ápice; parede celular com grandes trigônios e espessamentos intermédios, e anfigastros profundamente bífidos (Jovet-Ast 1953). Esporófito freqüente. Epífila, ocorrendo em grandes populações, em ambientes sombreados e úmidos no interior da mata.

Distribui-se na América Tropical. No Brasil é encontrada nos Estados: AM, SP (Yano 1995). Primeiro registro para a região Nordeste.

Diplasiolejeunea cobrensis Gottsche ex Steph., Spec. Hepat. 5: 923. 1916.

Fig.15-18.

Descrição: Reyes (1982).

Material examinado: BRASIL. Pernambuco: Cabo de Sto. Agostinho, Reserva Ecológica do Gurjaú, 1/IX/2001, galho, S.R. Germano (UFP 31499a; UFP 31501a).

Comentários: caracteriza-se pelos anfigastros estreitos, ca. 1-1,5 vezes a largura do caulídio, profundamente bífidos e parede celular com trigônios conspícuos. Esporófito frequiente. Epífita de galhos, 

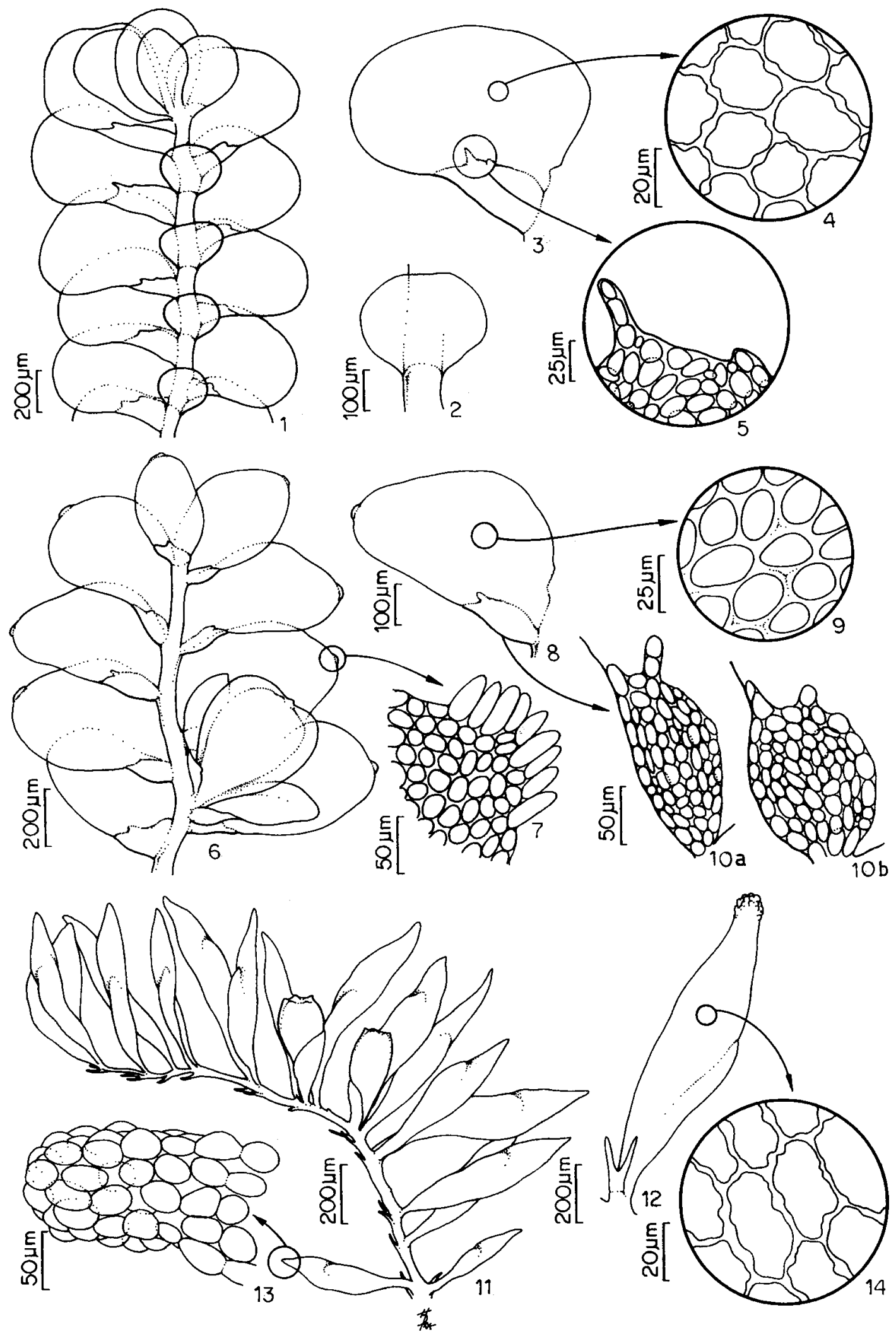

Figuras 1-5. Archilejeunea auberiana (Mont.) A. Evans. 1. Aspecto geral do gametófito. 2. Anfigastro. 3. Filídio destacando o lóbulo ventral. 4. Células centrais do filídio. 5. Dente apical do lóbulo. Figuras 6-10. Cololejeunea cardiocarpa (Mont.) A. Evans. 6. Aspecto geral do gametófito. 7. Ápice do filídio com células hialinas. 8. Filídio. 9. Células centrais do filídio. 10a, b. Lóbulo com detalhe do dente apical. Figuras 11-14. Colura greig-smithii Jovet-Ast. 11. Aspecto geral do gametófito. 12. Filídio e anfigastro. 13. Detalhe do ápice do filídio. 14. Células centrais do filídio. 
ocorrendo em pequenas populações, associada a outras hepáticas, no dossel externo de Tapirira guianensis Aubl.

Distribui-se na América Tropical. No Brasil é encontrada nos Estados: AL, BA, PR, RO (Bôas-Bastos \& Bastos 1998; Yano 1995). Primeiro registro para Pernambuco.

Harpalejeunea stricta (Lindenb. \& Gottsche) Steph., Hedwigia 27: 291. 1988.

Fig. 19-24.

Descrição: Schuster (1980).

Material examinado: BRASIL. Pernambuco: Cabo de Santo Agostinho, Reserva Ecológica do Gurjaú, 1/XI/2000, folha, S.R. Germano (UFP 31134d; UFP 31136d; UFP 31140b; UFP 31147e; UFP 31161d); ibid. 1/IX/2001, galho, S.R. Germano (UFP 31484b); ibid. 1/IX/2001, tronco vivo, S.R. Germano (UFP 31497a; UFP 31512c); ibid. 1/IX/2001, base de tronco vivo, S.R. Germano (UFP 31514c); ibid. 1/IX/2001, ramo, S.R. Germano (UFP 31516d); ibid. 1/IX/2001, galho, S.R. Germano (UFP 31522c); ibid. 1/IX/2001, tronco vivo, S.R. Germano (UFP $315571 \mathrm{~b}$; UFP 315572a); ibid. 19/X/2000, base de tronco vivo, S.R. Germano (UFP 32820a).

Comentários: espécie próxima a Harpalejeunea ovata Hook., diferindo desta por apresentar ápice curto, acuminado, recurvado, usualmente terminando em 1-3 células e perianto de quilhas lisas. Ocasionalmente encontrada com esporófito. Epífila e epífita de tronco, ramos e galhos de Tapirira guianensis Aubl. e Protium heptaphyllum Mart, ocorrendo em pequenas e grandes populações, tanto em locais sombreados, como naqueles de maior exposição.

Distribui-se na América Tropical e África. No Brasil é encontrada nos Estados: AC, BA, PA, RJ (Lisboa \& Ilkiu-Borges 1995; Bôas-Bastos \& Bastos 1998; Oliveira e Silva \& Yano 1998; Gradstein \& Costa 2003). Primeiro registro para Pernambuco.

Lejeunea caespitosa Lindenb. ex G.L. \& Nees, Syn. Hepat.: 382. 1845.

Fig. 25-28.

Descrição: Schuster (1980).

Material examinado: BRASIL. Pernambuco: Cabo de Sto. Agostinho, Reserva Ecológica do Gurjaú, 19/X/2000, base de tronco vivo, S.R. Germano (UFP 32801b); ibid. 1/XI/2000, folha, S.R. Germano (UFP 31131d; UFP 31145c; UFP 31146c; UFP 31153a).

Comentários: caracteriza-se pelo gametófito pequeno, verde-claro e translúcido; anfigastros pequenos, 1,0-1,5 vezes a largura do caulídio, 3-4 células de largura na base, distanciados entre si por um grande sinus, em forma de $\mathrm{U}$, com dentes em ambos os lados. Espécie de morfologia muito variada, apresentando lóbulos polimórficos, eventualmente reduzidos. Epífila e epífita de troncos, ocorrendo em pequenas populações, associada a outras hepáticas. Esporófito freqüente.

Anfitropical. No Brasil é encontrada nos Estados: AC, PA, RJ, SP (Schäfer-Verwimp \& Giancotti 1993; Oliveira e Silva \& Yano 1998). Primeiro registro para a região Nordeste.

Lejeunea monimiae (Steph.) Steph., Spec. Hepat. 5: 747. 1915.

Fig. 29-33.

Descrição: Reiner-Drehwald (2000).

Material examinado: BRASIL. Pernambuco: Cabo de Sto. Agostinho, Reserva Ecológica do Gurjaú, 1/XI/2000, folha, S.R. Germano (UFP 31144; UFP 31169h).

Comentários: caracteriza-se pelo gametófito verde-claro, transparente, com ramos do tipo Lejeunea abundantes. Lóbulos retangulares, muitas vezes reduzidos a poucas células; dente apical formado por uma célula inconspícua. Células basais e centrais com trigônios e espessamentos intermédios mais robustos do que o material das Misiones (Argentina), examinado por Dra. Elena Reiner Drewhald (com. pessoal). Perianto piriforme com quilhas crenuladas. Geralmente encontrada com esporófito. Epífila, ocorrendo em pequenas populações, associada a outras hepáticas, em ambiente sombreado no interior da floresta.

Distribui-se no Brasil e Argentina, sendo no Brasil encontrada em SC e SP (Reiner-Drehwald 2000). Primeiro registro para a região Nordeste.

Lejeunea quinqueumbonata Spruce, Trans. \& Proc. Bot. Soc. Edinburg 15: 230. 1884.

Fig. 34-38.

Descrição: Reiner-Drehwald (2000).

Material examinado: BRASIL. Pernambuco: Cabo de Sto. Agostinho, Reserva Ecológica do Gurjaú, 2/VI/2000, tronco morto, S.R. Germano (UFP 32802); ibid. 1/XI/2000, tronco morto, S.R. Germano (UFP 32835c; UFP 32831b; UFP 32887c; UFP 32837c; UFP 32832a; UFP 32838c; UFP 32843b; UFP 32840a; UFP 32844a; ibid. 3/XI/2000, tronco morto, S.R. Germano (UFP 32842b; UFP 32839b; UFP 32841b). 

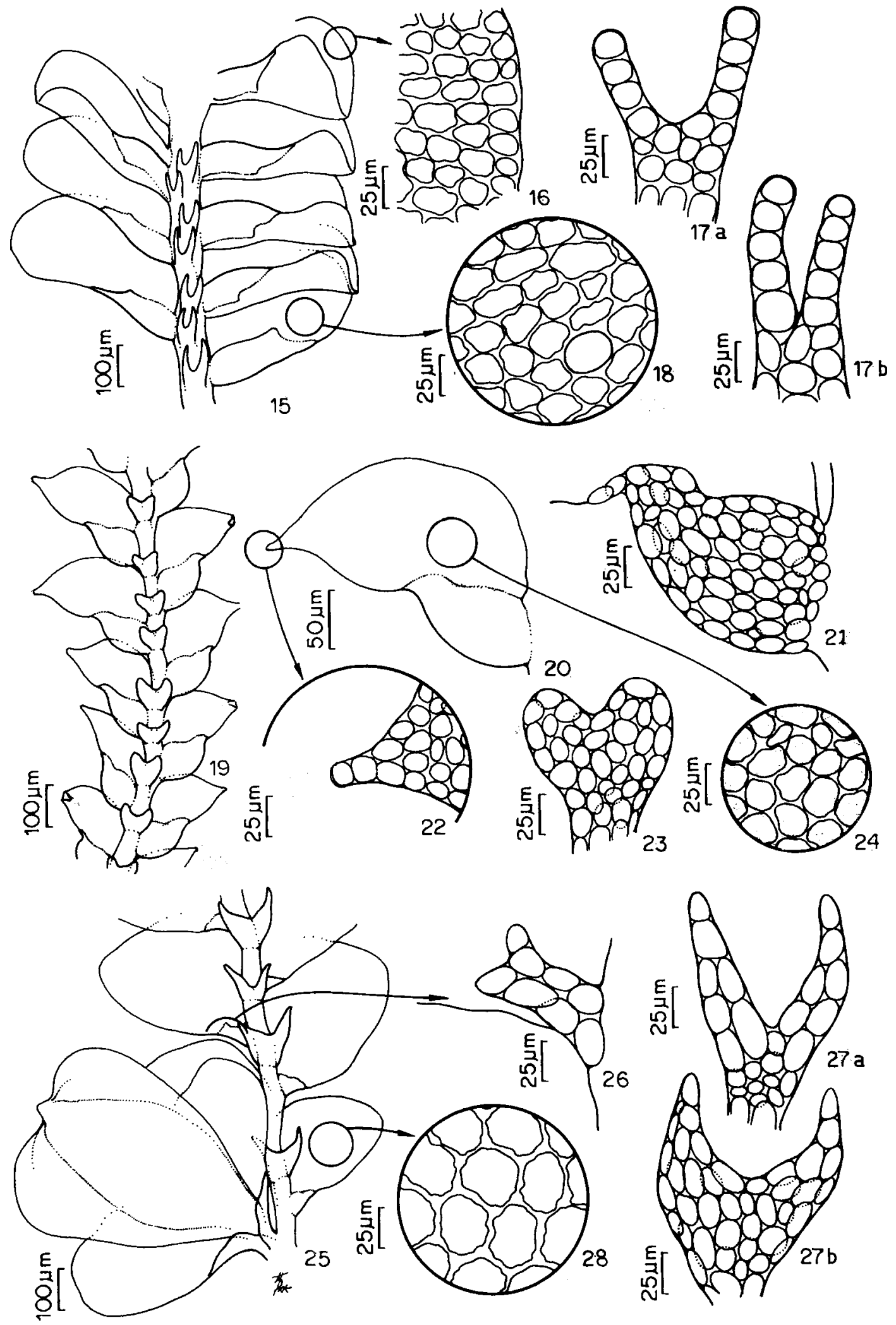

Figuras 15-18. Diplasiolejeunea cobrensis Gottsche ex Steph. 15. Aspecto geral do gametófito. 16. Células da margem do filídio. 17a, b. Anfigastro. 18. Células centrais do filídio. Figuras 19-24. Harpalejeunea stricta (Lindenb. \& Gottsche) Steph. 19. Aspecto geral do gametófito. 20. Filídio. 21. Lóbulo ventral. 22. Detalhe do ápice do filídio. 23. Anfigastro. 24. Células centrais do filídio. Figuras 25-28. Lejeunea caespitosa Lindenb. ex G.L. \& Nees. 25. Aspecto geral do gametófito - perianto. 26. Lóbulo reduzido. 27a, b. Anfigastro. 28. Células centrais do filídio. 


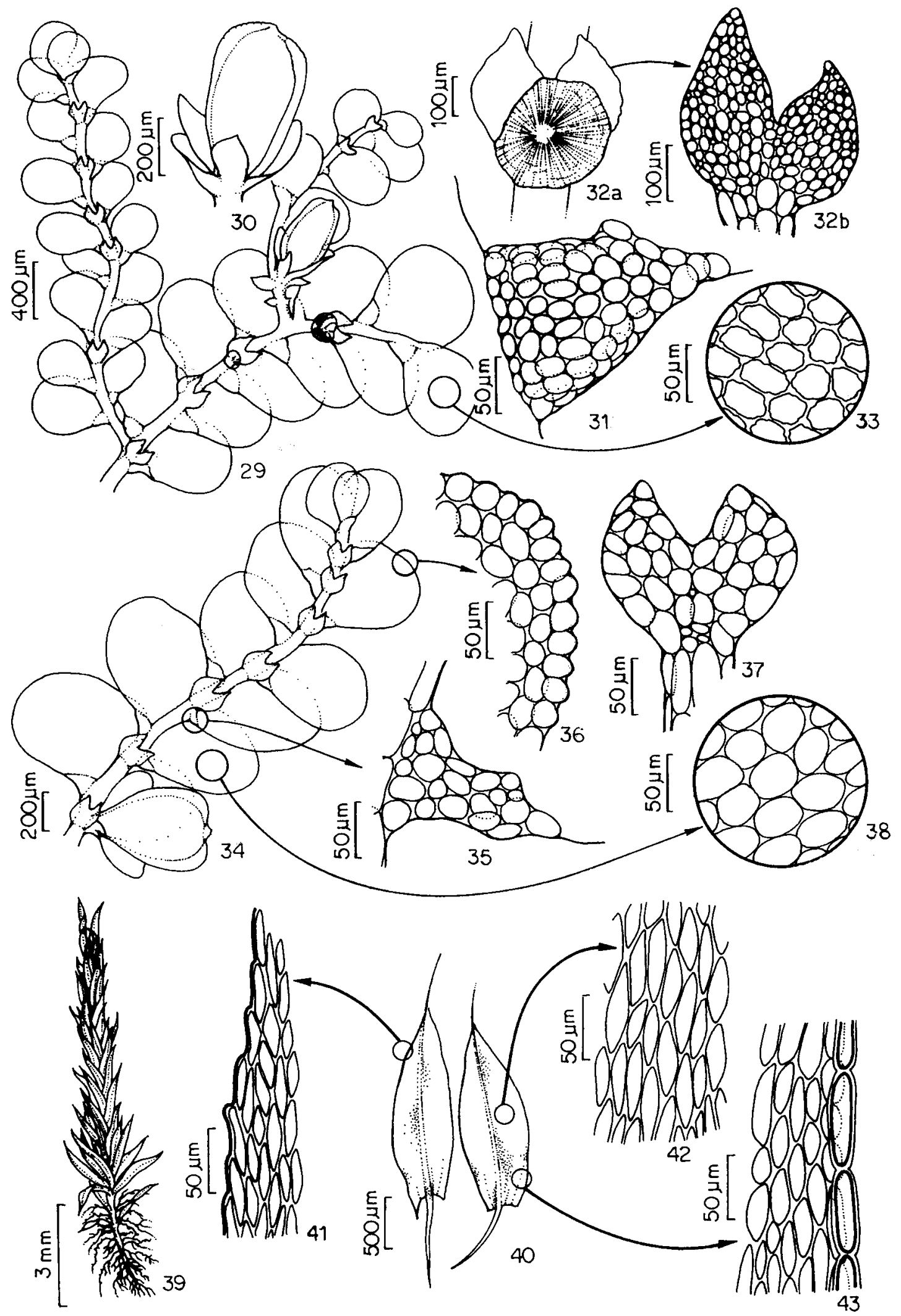

Figuras 29-33. Lejeunea monimiae (Steph.) Steph. 29. Aspecto geral do gametófito. 30 Perianto. 31. Lóbulo. 32a, b. Anfigastro. 33. Células centrais do filídio. Figuras 34-38. Lejeunea quinqueumbonata Spruce. 34. Aspecto geral do gametófito - perianto. 35. Lóbulo. 36. Células da margem do filídio. 37. Anfigastro. 38. Células centrais do filídio. Figuras 39-43. Bryum pabstianum C. Muell. 39 . Aspecto geral do gametófito. 40. Filídios. 41. Células da margem do filídio. 42. Células centrais do filídio. 43. Células basais do filídio. 
Comentários: caracteriza-se por apresentar filídios e brácteas periqueciais com margens crenuladas; células medianas do filídio com parede delgada e pequenos trigônios. Ocasionalmente pode ser encontrado perianto estipitado (Reiner-Drehwald 2000). Geralmente encontrada com esporófito. Epíxila, formando grandes tapetes, de cor verde claro brilhante a amarelada, em ambientes sombreados.

Distribui-se na América Tropical. No Brasil é encontrada nos Estados: AM, RJ, SP (Reiner-Drehwald 2000). Primeiro registro para a região Nordeste.

\section{BRYACEAE}

Bryum pabstianum C. Muell., Bot. Zeit. 13: 751. 1855.

Fig. 39-43.

Descrição: Ochi (1980).

Material examinado: BRASIL. Pernambuco: Cabo de Sto. Agostinho, Reserva Ecológica do Gurjaú, 5/IV/2000, solo plano, S.R. Germano (UFP 31837; UFP 31811; ibid. 17/X/2000, solo plano, S.R. Germano (UFP 32846; UFP 32847).

Comentários: espécie similar a Bryum subapiculatum Hampe, mas difere desta por ser sinóico, possuir filídios mais alongados-lanceolados, gemas rizoidais piriformes, alaranjadas e cápsula com opérculo delgado, curto-cônico (Ochi 1980). Terrícola, formando tapetes na margem e no interior da mata.

Distribui-se no Peru, Bolívia e Ilhas Malvinas. No Brasil é encontrada nos Estados: ES, GO, PR, RJ, RS, SC, SP (Yano 1981; 1989; 1995). Primeiro registro para a região Nordeste.

Os táxons são de ocorrência comum em floresta tropical úmida de terras baixas e apresentam distribuição Neo-, Pan-, ou Afro-americana. Na maioria são conhecidos para vários países da América Tropical, com exceção de Lejeunea monimiae, cuja distribuição é restrita ao sul da América do Sul, no Brasil e na Argentina. Algumas espécies, tais como Archilejeunea auberiana e Cololejeunea cardiocarpa são de ampla distribuição no Brasil sendo citadas pelo menos para quatro regiões geográficas. Por sua vez, Lejeunea quinqueumbonata e Colura greig-smithii são referidas pela primeira vez para o Nordeste, tendo sido assinaladas, anteriormente, apenas para alguns Estados das regiões Norte e Sul ou Sudeste.

A maioria dos representantes de Lejeuneaceae analisados ocorrem em locais melhor preservados, sombreados e úmidos, no interior da Reserva, ou próximos a fontes hídricas. Quanto aos microhábitats de ocorrência, as espécies apresentaram-se preferencialmente epífitas de troncos - A. auberiana, de galhos - D. cobrensis, ou ainda, de folhas - C. cardiocarpa. Apenas $L$. quinqueumbonata foi exclusivamente epíxila, ocorrendo em troncos de diferentes estádios de decomposição e Bryum pabstianum, terrícola, em ambientes expostos ao sol.

\section{Agradecimentos}

As autoras agradecem à Dra. Denise Pinheiro da Costa, do Jardim Botânico do Rio de Janeiro; à Dra. Olga Yano, do Instituto de Botânica de São Paulo, à Dra. M. Elena Reiner Drehwald, do Systematisch Geobotanisches Institut, University of Göttingen Alemanha, pela confirmação de algumas espécies; a CAPES, CNPq e WWF, pelo apoio financeiro.

\section{Referências bibliográficas}

Bôas-Bastos, S.B.V. \& Bastos, C.J.P. 1998. Briófitas de uma área de Cerrado no município de Alagoinhas, Bahia, Brasil. Tropical Bryology 15: 101-110.

FIDEM - Fundação de Desenvolvimento da Região Metropolitana do Recife. 1993. Monitoramento das Reservas Ecológicas da RMR. Governo do Estado de Pernambuco, Recife.

Germano, S.R. \& Pôrto, K.C. 1996. Floristic survey of epixylic bryophytes of an area remnant of the Atlantic Forest (Timbaúba, PE, Brazil) 1. Hepaticopsida (except Lejeuneaceae) and Bryopsida. Tropical Bryology 12: $21-28$.

Germano, S.R. \& Pôrto, K.C. 1998a. Briófitas epíxilas de uma área remanescente de Floresta Atlântica (Timbaúba, PE, Brasil). 2. Lejeuneaceae. Acta Botanica Brasilica 12(1): 53-66.

Germano, S.R. \& Pôrto, K.C. 1998b. Adições a brioflora do Estado de Pernambuco. Hoehnea 25(2): 121-131.

Gradstein, S.R. 1989. A key to the Hepaticae and Anthocerotae of Puerto Rico and the Virgin Islands. The Bryologist 92(3): 329-348.

Gradstein, S.R. 1994. Lejeuneaceae: Ptychantheae, Brachiolejeuneae. Flora Neotropica: Monograph 62: 1-216.

Gradstein, S.R. \& Costa, D.P. 2003. The Hepaticae and Anthocerotae of Brazil. Memoirs of the New York Botanical Garden 87: 1-318.

Heinrichs, J. \& Gradstein, S.R. 2000. A revision of Plagiochila sect. Crispatae and sect. Hypnoides (Hepaticae) in the Neotropics. I. Plagiochila disticha, P. montagnei and $P$. raddiana. Nova Hedwigia 70: 161-184.

Jovet-Ast, S. 1953. Le genre Colura. Hepatiques Lejeuneaceae, Diplasiae. Revue Bryologique et Lichénologique 22: 206-312. 
Lisboa, R.C.L. \& Ilkiu-Borges, A.L. 1995. Diversidade das briófitas de Belém (PA) e seu potencial como indicadoras de poluição urbana. Boletim do Museu Paraense Emílio Goeldi 11(2): 199-225.

Ochi, H. 1980. A revision of the Neotropical Bryoideae, Musci (First part). Journal of Faculty Education Tottori University 29(2): 45-154.

Oliveira e Silva, M.I.M.N. \& Yano, O. 1998. Ocorrências novas de briófitas para o Brasil. Revista Brasileira de Botânica 21(2): 125-134.

Pôrto, K.C. 1990. Bryoflores d'une forêt de plaine et d'une forêt d'altitude moyenne dans l'État de Pernambuco (Brésil). Analyse floristique. Cryptogamie, Bryologie Lichénologie 11(2): 109-161.

Pôrto, K.C. \& Germano, S.R. 2002. Biodiversidade e importância das briófitas na conservação dos ecossistemas naturais de Pernambuco. Pp.125-152. In: M. Tabarelli \& J.M.C. Silva (orgs.). Diagnóstico da Biodiversidade de Pernambuco. SECTMA - Secretaria de Ciência, Tecnologia e Meio Ambiente, Recife.

Pôrto, K.C.; Belo, M.M.L.; Fonseca, E.R. \& Silva, E.C. 1993. Brioflora da Reserva do Gurjaú (Cabo - PE). Biologica Brasilica 5(1/2): 27-42.

Pôrto, K.C.; Germano, S.R. \& Oliveira, S.M. 2000. New records of bryophytes Pernambuco State, Brazil. Tropical Bryology 18: 107-114.

Pôrto, K.C.; Gradstein, S.R.; Yano, O.; Germano, S.R. \& Costa, D.P. 1999. New and interesting records of Brazilian bryophytes. Tropical Bryology 17: 19-45.

Reiner-Drehwald, M.E. 2000. Las Lejeuneaceae (Hepaticae) de Misiones, Argentina. VI Lejeunea y Taxilejeunea. Tropical Bryology 19: 81-131.
Reyes, D.M. 1982. El género Diplasiolejeunea en Cuba. Acta Botanica Academiae Scientiarum Hungarica 28(1-2): 145-180.

Sá, P.S.A. \& Pôrto, K.C. 1996. Novos registros de Hepaticopsida para Pernambuco. Revista Nordestina de Biologia 11(1): 37-43.

Schäfer-Verwimp, A. \& Giancotti, C. 1993. New interesting records of Brazilian bryophytes, IV. Hikobia 11: 285-292.

Schuster, R.M. 1980. The Hepaticae and Anthocerotae of North America. v. IV. Columbia University Press, New York.

Tixier, P. 1985. Contribution à la connaissance des Cololejeunoideae. Bryophytorum Bibliotheca 27: 298-351.

Valdevino, J.A.; Sá, P.S.A. \& Pôrto, K.C. 2002. Musgos pleurocárpicos de mata serrana em Pernambuco, Brasil. Acta Botanica Brasilica 16(2): 161-174.

Vital, D.M. \& Visnadi, S.R. 1994. Bryophytes of Rio Branco Municipality, Acre, Brazil. Tropical Bryology 9: 69-74.

Yano, O. 1981. A checklist of Brazilian mosses. The Journal of the Hattori Botanical Laboratory 50: 279-456.

Yano, O. 1984. Checklist of Brazilian liverworts and hornworts. The Journal of the Hattori Botanical Laboratory 56: 481-548.

Yano, O. 1989. An additional checklist of brazilian bryophytes. The Journal of the Hattori Botanical Laboratory 66: 371-434.

Yano, O. 1995. A new additional annotated checklist of brazilian bryophytes. The Journal of the Hattori Botanical Laboratory 78: 137-182.

Yano, O. 1996. A checklist of the Brazilian Bryophytes. Boletim do Instituto de Botânica 10: 47-232.

Yano, O. \& Andrade-Lima, D. 1987. Briófitas no Nordeste brasileiro: Estado de Pernambuco. Revista Brasileira de Botânica 10: 171-181. 